\title{
The cryogenic control system of BEPCII
}

Gang $\mathrm{Li}^{1,2^{*}} \mathbb{D}$, Rui $\mathrm{Ye}^{1}$, Mingjing Sang ${ }^{1}$, Shaopeng $\mathrm{Li}^{1,2}$, Zhuo Zhang ${ }^{1}$, Jiehao Zhang ${ }^{1}$, Ruixiong Han ${ }^{1}$, Ge Lei ${ }^{1,2}$ and Rui Ge $e^{1,2}$

\author{
* Correspondence: li75gang@ihep. \\ ac.cn \\ ${ }^{1}$ Laboratory of Particle Acceleration \\ Physics \& Technology, Institute of \\ High Energy Physics, Chinese \\ Academy of Sciences, Beijing \\ 100049, China \\ ${ }^{2}$ University of Chinese Academy of \\ Science, Beijing 100049, China
}

\begin{abstract}
The BEPCII project is for upgrading the Beijing Electron Positron Collider to reach a higher luminosity. The cryogenic system has been firstly designed and deployed in BEPCII, which provides cooling capacity for SRF cavities, SCQ and SSM. The cryogenic control system consists of Siemens PLC for the refrigerator control and "PLC + IOC" for superconducting device control. The BEPCII machine commissioning with beam started in October 2006. This paper describes the design and the development of the cryogenic control system.
\end{abstract}

Keywords: BEPCII, Cryogenic control system, PLC, IOC

\section{Introduction}

BEPCII is a two-ring $\mathrm{e}^{+}$and $\mathrm{e}^{-}$collider running in the tau-charm energy region $(\mathrm{Ecm}=$ 2.0-4.2 GeV), which, with a design luminosity of $1 \times 10^{33} \mathrm{~cm}^{-2} \mathrm{~s}^{-1}$ at the beam energy of $1.89 \mathrm{GeV}$, is an improvement of a factor of 100 over its successful predecessor, BEPC [1]. The upgrade uses the existing tunnel, some major infrastructure items, and some of the old magnets. The $202 \mathrm{~m}$ long LINAC of the new machine can accelerate electrons and positrons up to $1.89 \mathrm{GeV}$ with a positron injection rate of $50 \mathrm{~mA} / \mathrm{min}$. The collider consists of two $237.5 \mathrm{~m}$ long storage rings, one for electrons and one for positrons. The machine will also provide a high flux of synchrotron radiation at a beam energy of $2.5 \mathrm{GeV}$. BESIII at the BEPCII accelerator is a major upgrade of BESII at the BEPC for the studies of hadron physics and $\mathrm{T}$-charm physics with the highest accuracy achieved until now.

Compared to the BEPC and BESII, the performance of BEPCII and BESIII have been greatly improved, and some superconducting technologies have been used. For the BEPCII, the collider has two Superconducting Radio Frequency (SRF) cavities used to accelerate the beam, two superconducting quadrupole (SCQ) magnets at the colliding point to focus the beam. Figure 1 shows the schematic and photograph view of the $500 \mathrm{MHz}$ SRF cavity. The cylindrical core of the BESIII detector consists of a heliumbased multilayer drift chamber (MDC), a plastic scintillator time-of-flight system (TOF), and a CsI (Tl) electromagnetic calorimeter (EMC), which are all enclosed in a superconducting solenoidal magnet (SSM) providing a $1.0 \mathrm{~T}$ magnetic field with $4.5 \mathrm{~K}$

(c) The Author(s); licensee Springer on behalf of EPJ 2021 Open Access This article is licensed under a Creative Commons Attribution 4.0 International License, which permits use, sharing, adaptation, distribution and reproduction in any medium or format, as long as you give appropriate credit to the original author(s) and the source, provide a link to the Creative Commons licence, and indicate if changes were made. The images or other third party material in this article are included in the article's Creative Commons licence, unless indicated otherwise in a credit line to the material. If material is not included in the article's Creative Commons licence and your intended use is not permitted by statutory regulation or exceeds the permitted use, you will need to obtain permission directly from the copyright holder. To view a copy of this licence, visit http://creativecommons.org/licenses/by/4.0/ 


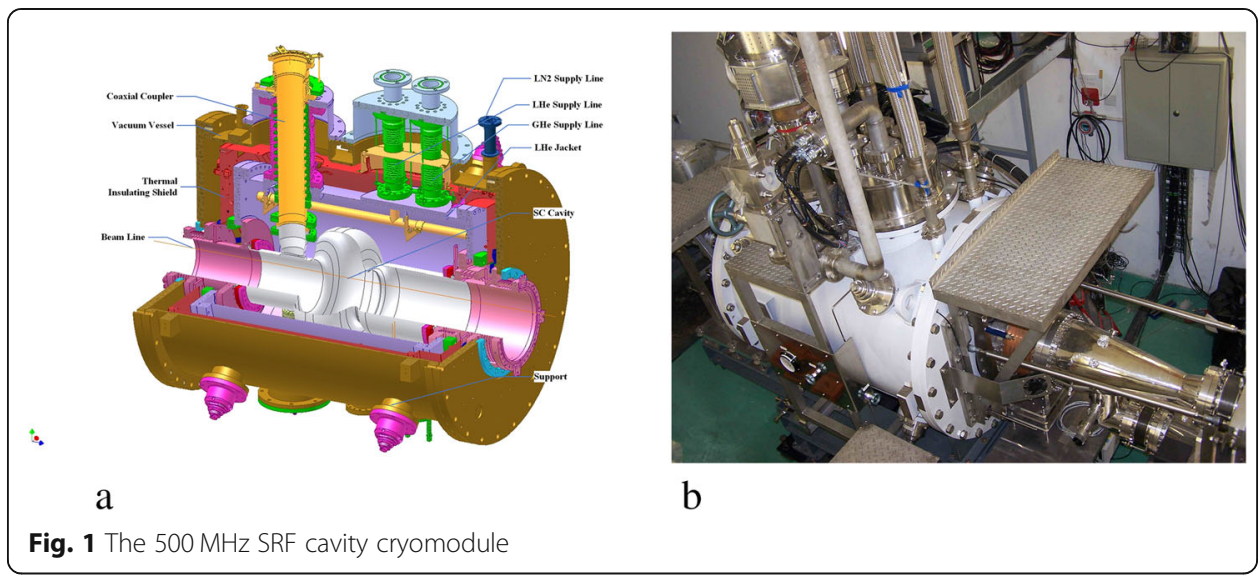

working temperature. Figure 2 shows the schematic view of the BESIII detector and photograph with the magnet iron open and end caps exposed.

\section{Cryogenic system}

To keep the stable operation of superconducting devices (two $500 \mathrm{MHz}$ superconducting RF cavities, two superconducting interaction quadrupole magnets, and one superconducting detector solenoid), the cryogenic system with totally capacity of $1 \mathrm{~kW}$ at $4.5 \mathrm{~K}$ was designed. The BEPCII cryogenic system consists of two cryogenic loops, one is SRF cavity cooling loop and another is SC magnets cooling loop. Each loop has a 500 W@4.5 K helium refrigerator, a liquid nitrogen tank, two helium gas tanks, a purification unit, and a corresponding cryogenic distribution system. The process flow diagram of SRF cavity cryogenic loop system is shown in Fig. 3a. The SRF cavity is immersed in saturated liquid helium. In stable operation mode, the liquid level is controlled at $91 \pm 1 \%$ and the pressure fluctuation is \pm 3 mbar. The process flow diagram of superconducting magnet cryogenic loop system is shown in Fig. 3b. The two SCQ magnets are cooled by supercritical helium and SSM is cooled with the forced two-phase helium at $4.5 \mathrm{~K}$. The construction of the cryogenic system for the BEPCII was completed in June 2006 and has been operated stably for 14 years. Figure 4 shows the layout of the cryogenic building and devices. A detailed control logic and interlocks, which are related to different operating modes of each device in the BEPCII cryogenic system, were designed and developed.

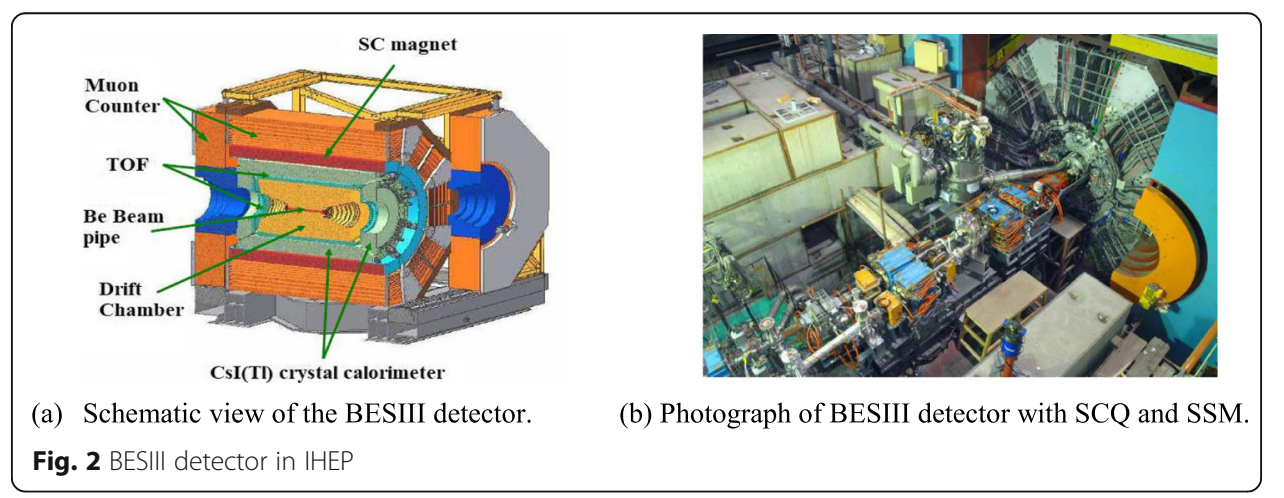




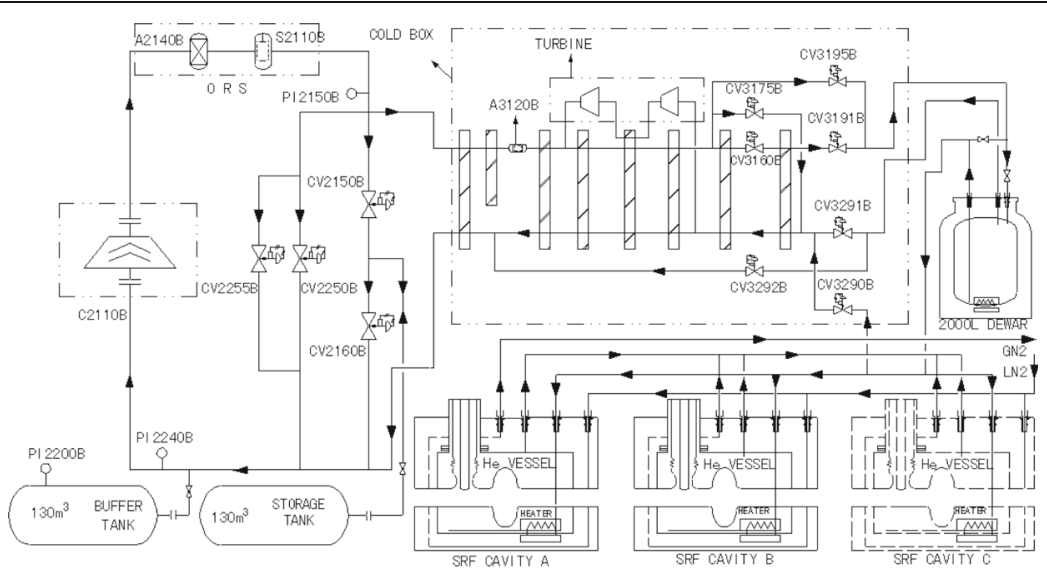

(a) Brief process flow diagram of $500 \mathrm{MHz}$ SRF cavity helium cryogenic loop system

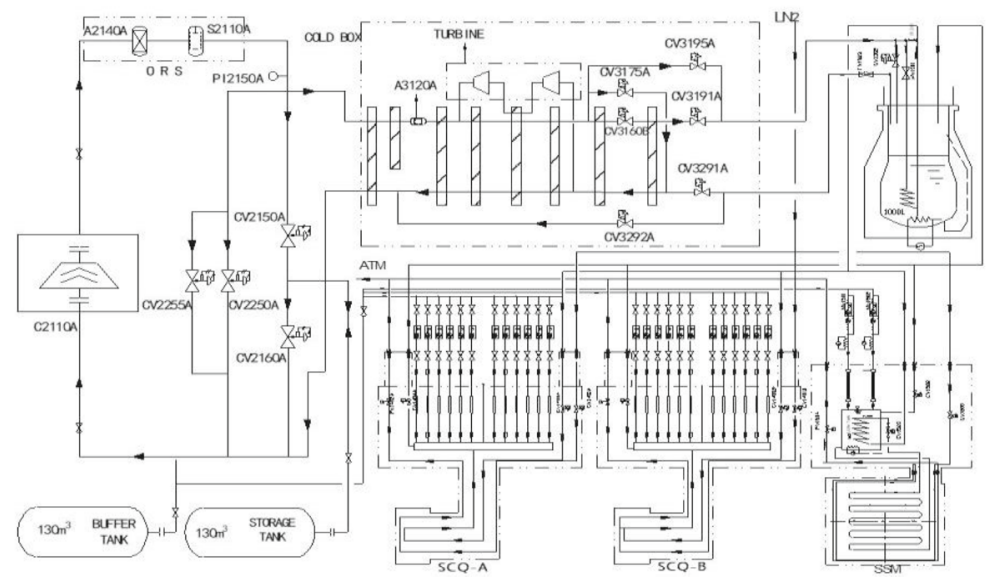

(b) Brief process flow diagram of superconducting magnet helium cryogenic loop system

Fig. 3 The process flow diagrams of BEPCII cryogenic loop system

\section{Cryogenic control system}

The cryogenic control system is mainly composed of refrigerator control and superconducting device control according to the front-end devices. The control system of refrigerator is a turnkey project, while the superconducting device control system, which covers the valve box, Dewar and conveying pipeline, is designed and developed by IHEP [2]. The structure of the cryogenic control system is shown in Fig. 5.

The refrigerator control system adopts Siemens S7-400/PLC, Profibus field bus and WinCC to control the front-end equipment, which has six working modes: refrigeration mode, mixed mode, liquefaction mode, warm-up mode, failure mode and off mode. Most of the time, the refrigeration mode is used to provide enough cooling for the device like SSM, SRF Cavity and SCQ. The synoptic display of the refrigerator control is shown in Fig. 6.

The control system of BEPCII is designed and built on EPICS (Experimental Physics and Industrial Control System), which is a set of open source software tools, libraries and applications developed collaboratively and used worldwide to create distributed soft real-time control systems for scientific instruments such as particle accelerators, telescopes and other large scientific experiments (http://www.aps.anl.gov/epics/about. 


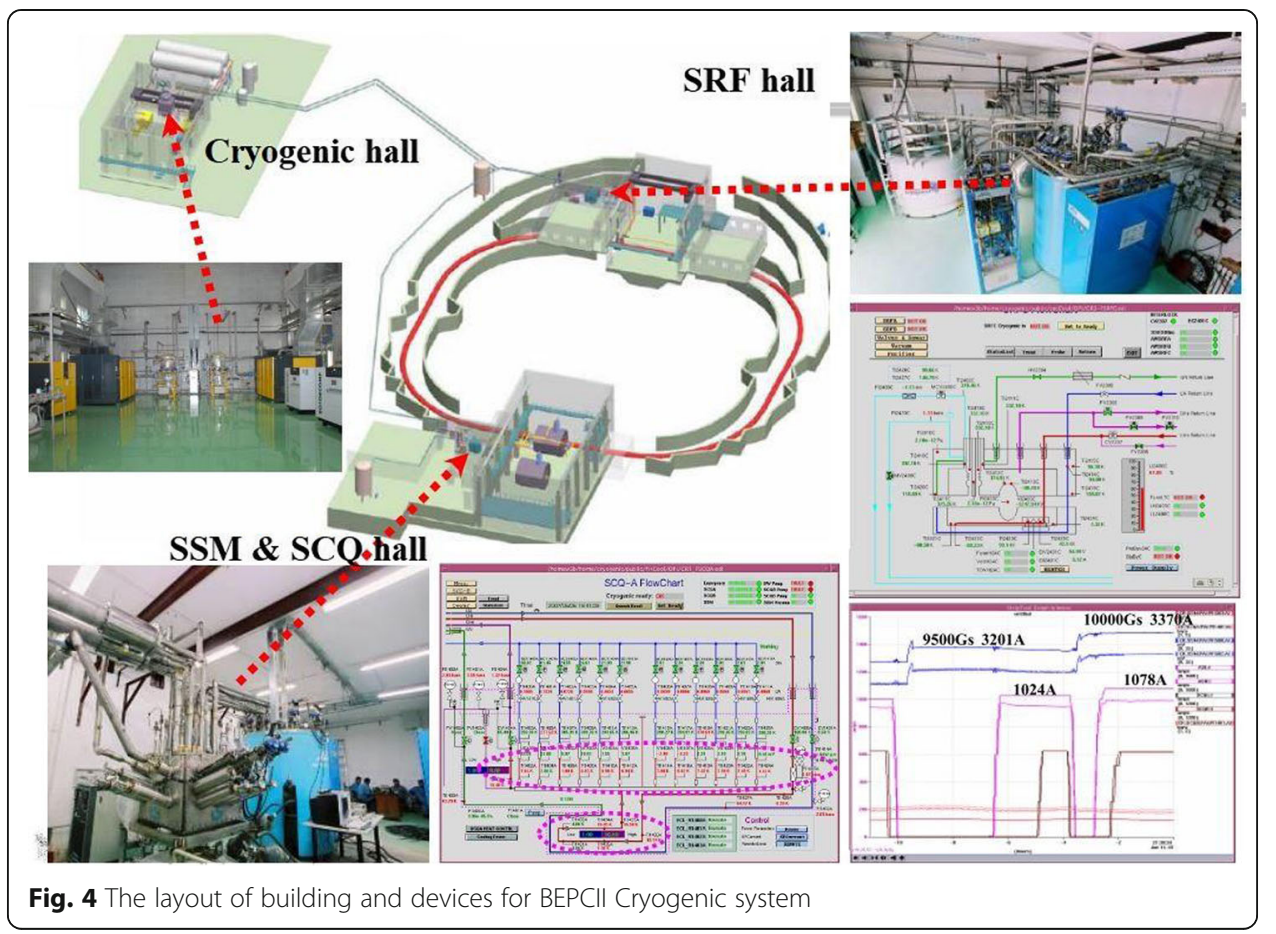

php) EPICS is a distributed control software based on the standard model of the clientserver, as shown in Fig. 7. The EPICS software structure includes the operator module (OPI) on the client side, the input and output control module (IOC) on the server side and the channel access module (CA).

To facilitate integration and maintenance operation, superconducting devices control system is also designed and developed based on EPICS, using Allen-Bradley PLC, ControlNet and VME IOCs. The PLCs and IOCs communicate through the ControlNet bus. The IOCs are MVME PowerPC controllers running VxWorks operating system in VME crates.

\section{Design of double-layer control}

If the cryogenic system has fault, it will take a long time to recover. Therefore, once the cryogenic system operates, it is expected to run continuously and steadily. It is very important to design a reliable control system for this reason.

During the commissioning and operation of the cryogenic system, it is often necessary to modify some of the control flow and control logic to optimize the cryogenic process according to the working conditions of superconducting device. If the traditional PLC control method is adopted, the newly compiled application software must be loaded into the PLC processor. After the PLC processor is powered on again, the new control flow and control logic can take effect, which will cause shutdown of the cryogenic system, and even the quench of superconducting device. It is required that the control system has more flexibility and can modify the control logic in real time according to the new requirements.

Generally, the applications program running in the PLC must work continuously, even momentary interruptions can't be tolerated whereas a short interruption can be 


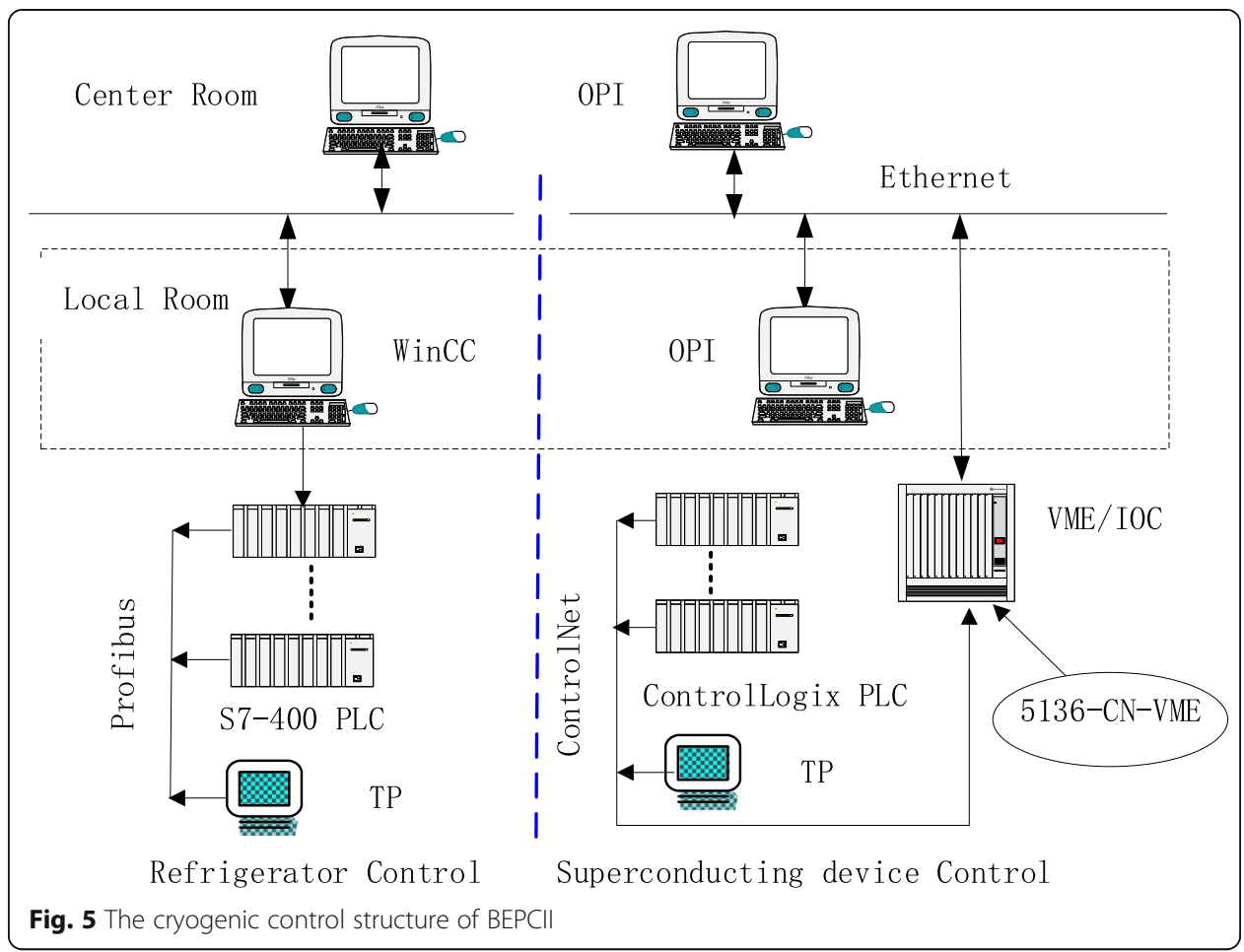

tolerated in the IOC side. The superconducting device control system adopts "EPICS + PLC" double-layer structure, see Fig. 8. The control logic and control flow are in EPIC $\mathrm{S}-\mathrm{IOC}$, and the interlock protection logic is in PLC. In this way, the flexibility of EPICS and the reliability of front-end PLC are fully applied, which makes EPICS and PLC independent and unified [3].

\section{Design of PID closed loop}

To ensure the smooth operation of the cryogenic system, the control system has designed many PID algorithms to adjust and control process variables such as liquid level, pressure and temperature. Figure 9 is a typical PID closed-loop structure diagram, where $\mathrm{AI}$ is the process variable from the temperature sensor, PID is the closed-loop regulation algorithm, and $\mathrm{AO}$ is the output signal of the control valve.

The cryogenic system has several operational modes including cooldown, normal operation, warmup and quench recovery. During the different operational modes, the different control logic, and control loops are used to control the actuator, the heater and so on. For example, in the cooldown mode, the Liquid helium inlet valve is controlled by PID algorithm to keep the temperature, while in the normal operation, the same valve is controlled by PID to maintain the liquid level. The "one output and two PIDs" closed-loop is designed, as shown in the Fig. 10.

\section{Safety interlock of the key device}

The cryogenic control system should not only guarantee the long-term stable operation of superconducting devices (SRF, SCQ and SSM), but also manage the safety of the 


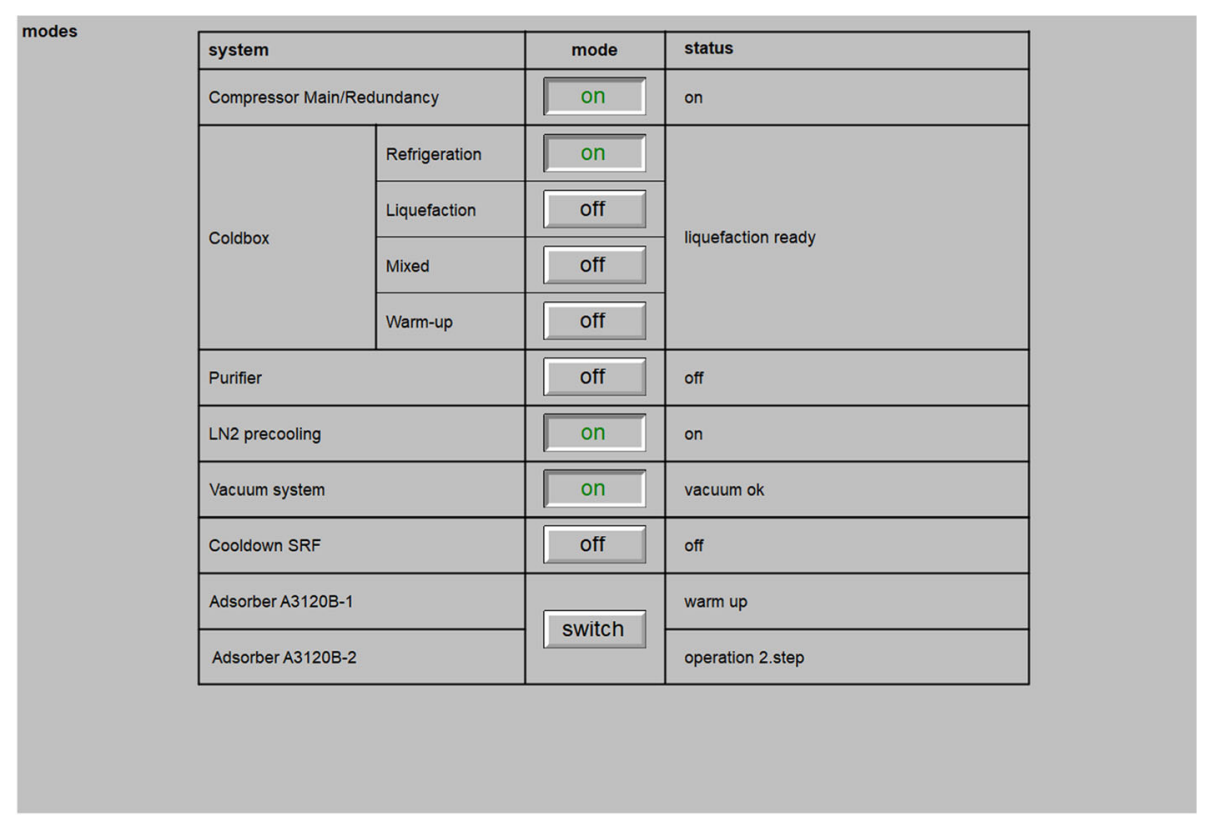

(a) selection of working mode

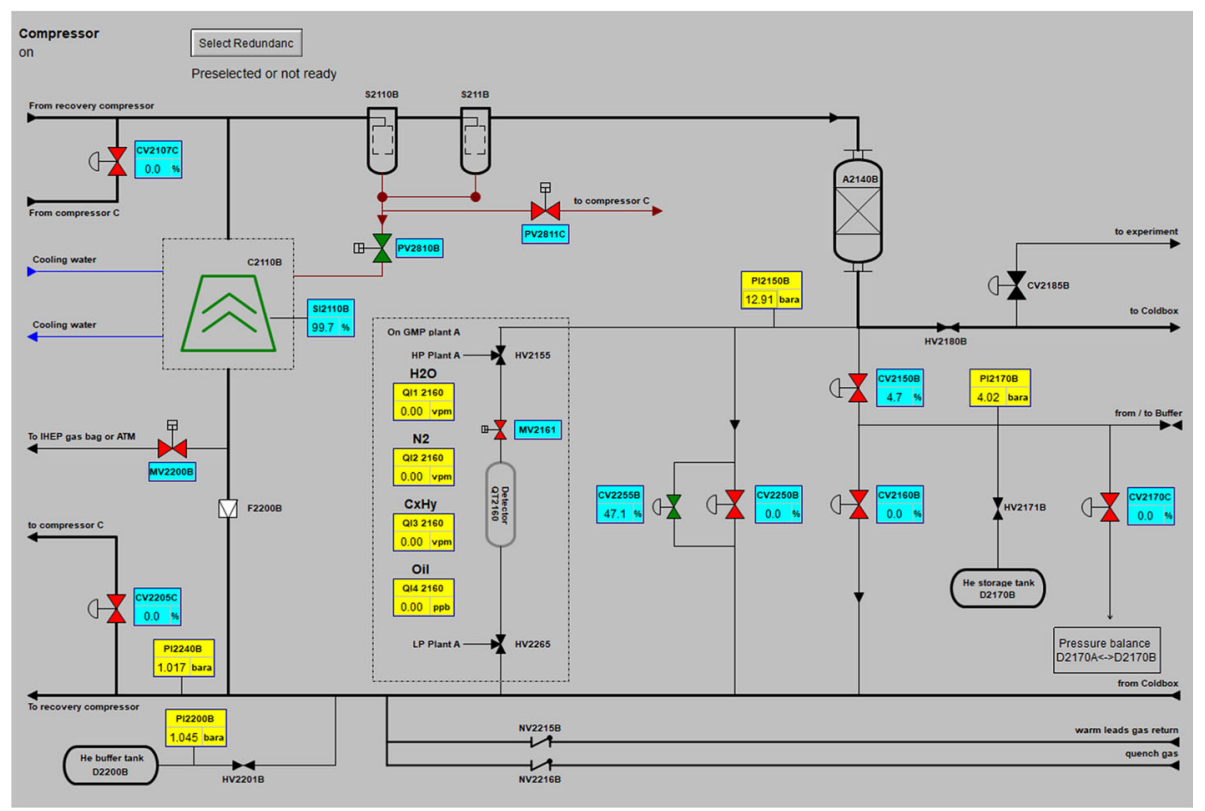

(b) the OPI of the refrigerator

Fig. 6 The refrigerator control system

equipment in the case of failure. In order to protect the key devices from damage, a lot of interlock logics need to be taken into account to force actuators at a safe position.

If the liquid helium does not begin to accumulate in the SRF cavity vessel, it is strictly forbidden to turn on the level gauge; if the liquid level the SRF cavity vessel is low, the heater power must be shut down immediately to avoid burning the heater; If SCQ or SSM is quenched, the flow controller is forced to open to the maximum opening, and the current lead is cooled by the maximum flow helium to prevent the current lead from burning out. The interlock actions in PLC are performed automatically as soon as 


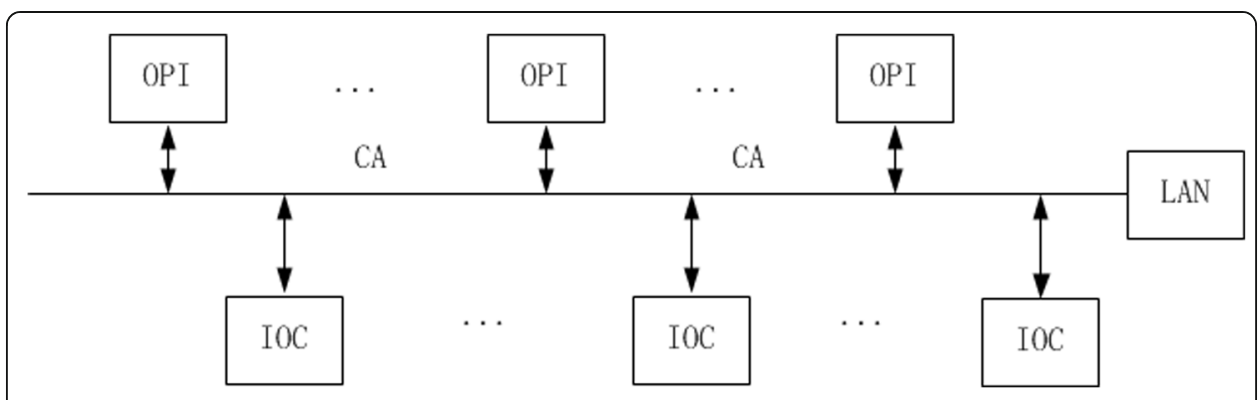

Fig. 7 The Software Structure of EPICS

one of the interlock conditions is triggered. However, the reset of the interlock function must be confirmed by the operator.

\section{Generation of cryogenic ready signal}

The operation of the cryogenic system provides reliable support for superconducting equipment. The control system automatically checks status information of the cryogenic system and generates a ready signal. Only when the superconducting device receives ready signal from the cryogenic system, it can be powered on. Figure 11 shows that the cryogenic ready signal consists of two parts, one part is "Epics_Ready" composed of process variables from the cryogenic system, the other part is "Set_Ready" signal from the operator. When both "Epics-Ready" and "Set-Ready" are valid, the "cryogenic ready" signal can be sent out. Any signal alarm or out of range will disable "cryogenic ready" signal, and the superconducting equipment must be powered off immediately.

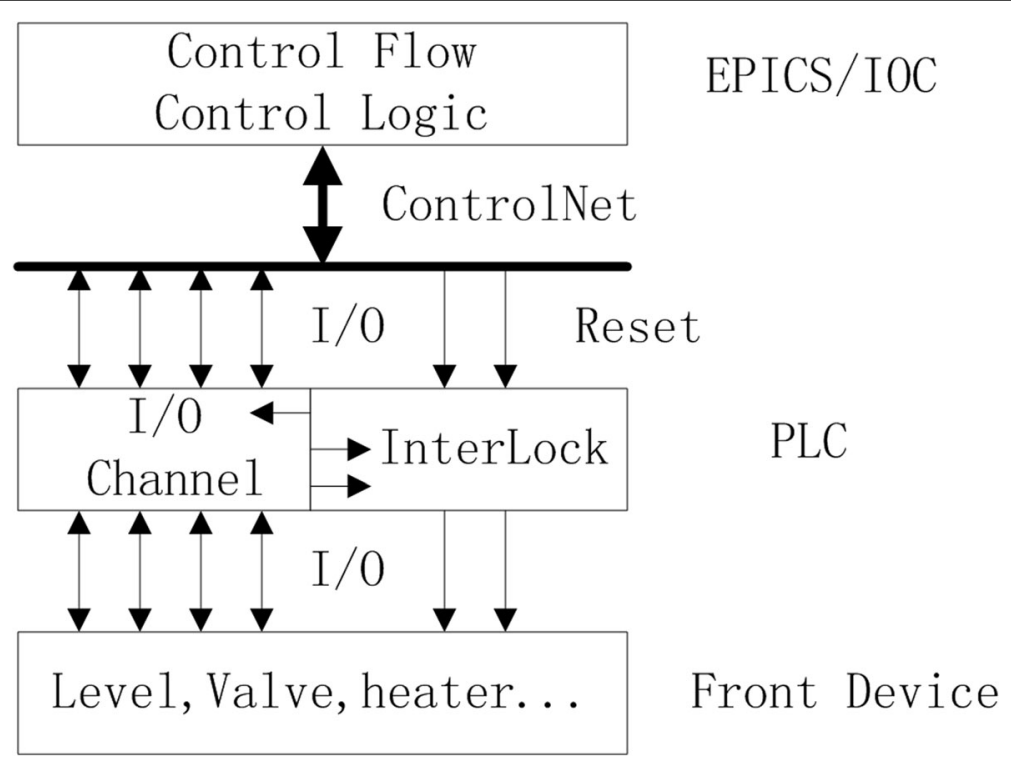

Fig. 8 The superconducting device control of "EPICS + PLC" double-layer structure 

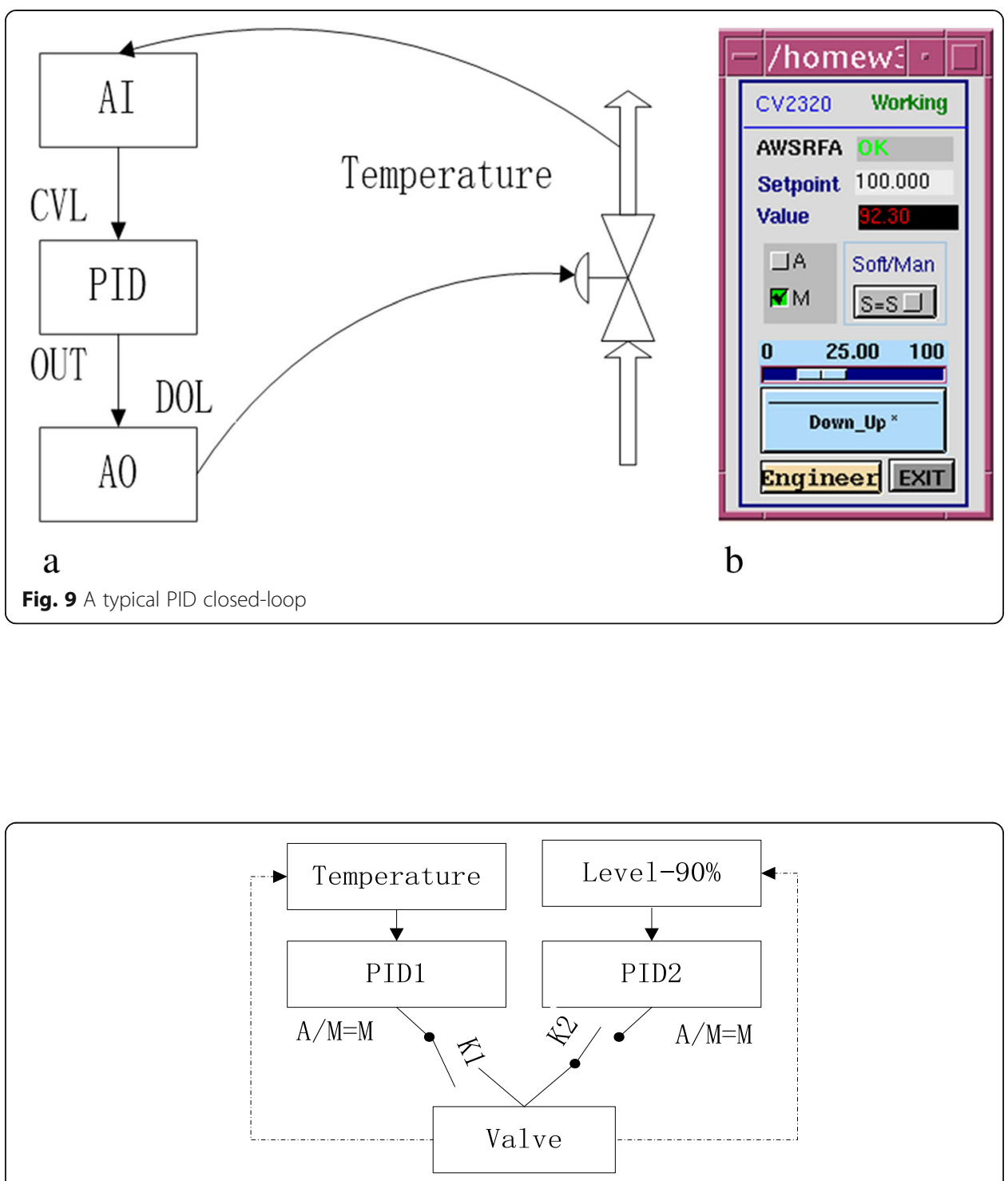

(a) Two PIDs closed-loop

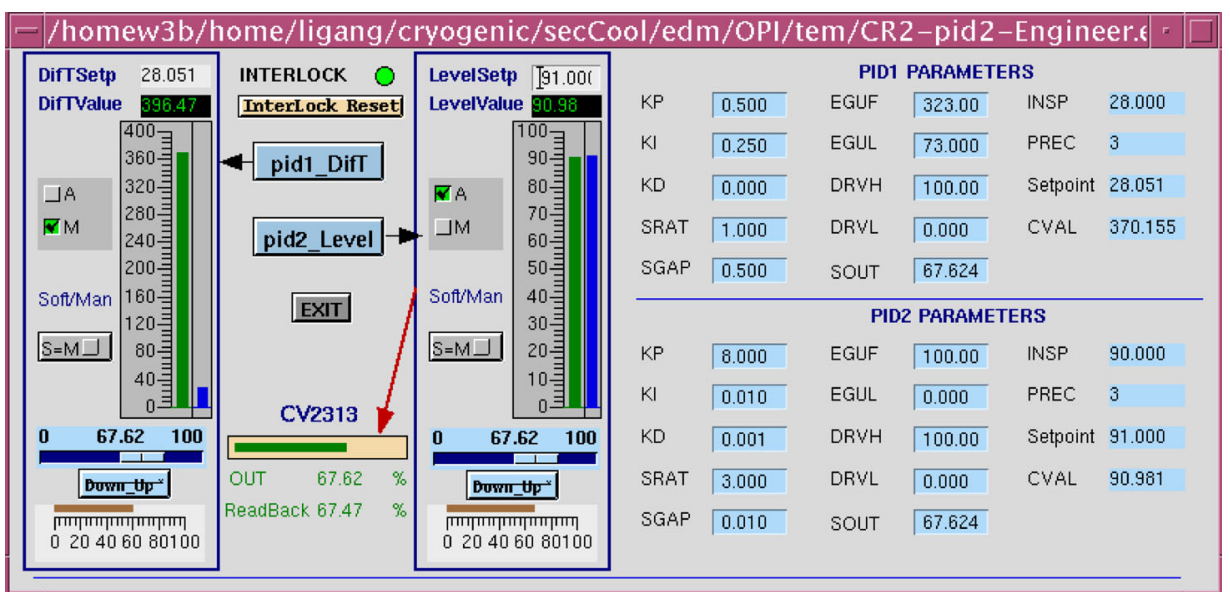

(b) the synoptic display of two PIDs closed-loop

Fig. 10 One output and two PIDs 


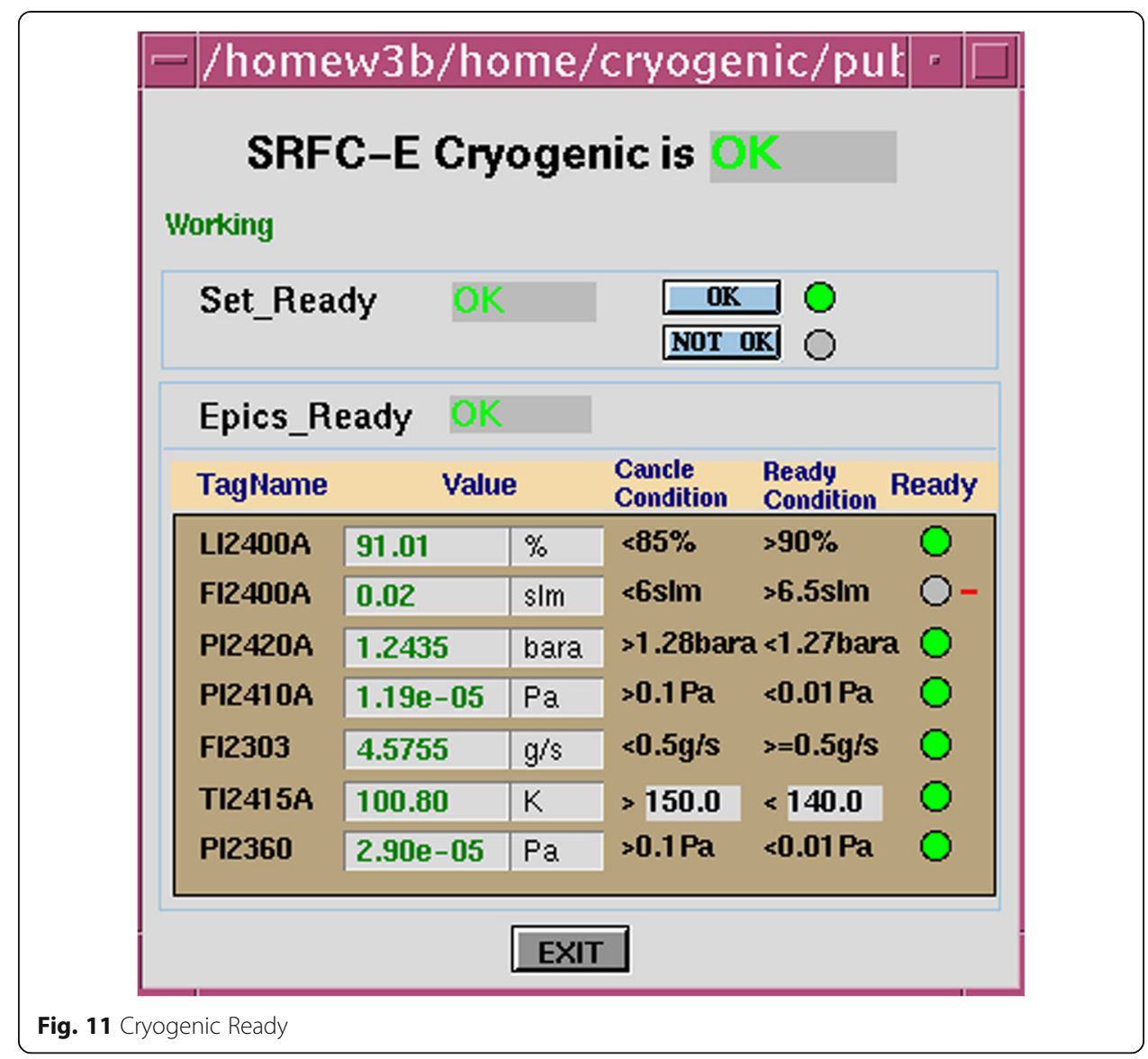

\section{Power compensation}

The SRF cavity is immersed in saturated liquid helium. When the high power is fed into the SRF cavity, a large amount of heat is generated instantaneously. Therefore, the power of the electrical heater is used to compensate the dynamical thermal load of the SRF Cavity to maintain a constant thermal load operation of the cryogenic system. The output power formula of electrical heaters is as follows:

$$
P_{\text {Heater }}=P_{\text {State }}+P_{\mathrm{RF}}
$$

where $\mathrm{P}_{\text {Heater }}$ represents the actual output power of the electrical heater; $\mathrm{P}_{\text {State }}$ stands for the static power of liquid helium vessel; $\mathrm{P}_{\mathrm{RF}}$ means the dynamic load power from the RF system. When $\mathrm{P}_{\mathrm{RF}}$ increases or decreases power, $\mathrm{P}_{\text {Heater }}$ simultaneously reversely decreases or increases the output power of the electrical heater, keeping the thermal load balance in the cryo-module all the time.

\section{Current leads control of SSM and SCQ}

The two SCQ magnets are cooled by the supercritical helium and SSM is cooled with the forced two-phase helium at $4.5 \mathrm{~K}$. The control of SSM and SCQ current leads must meet the following rules: Helium flow through current leads must be high enough to avoid introducing heat into the magnet; Helium temperature should be greater than 

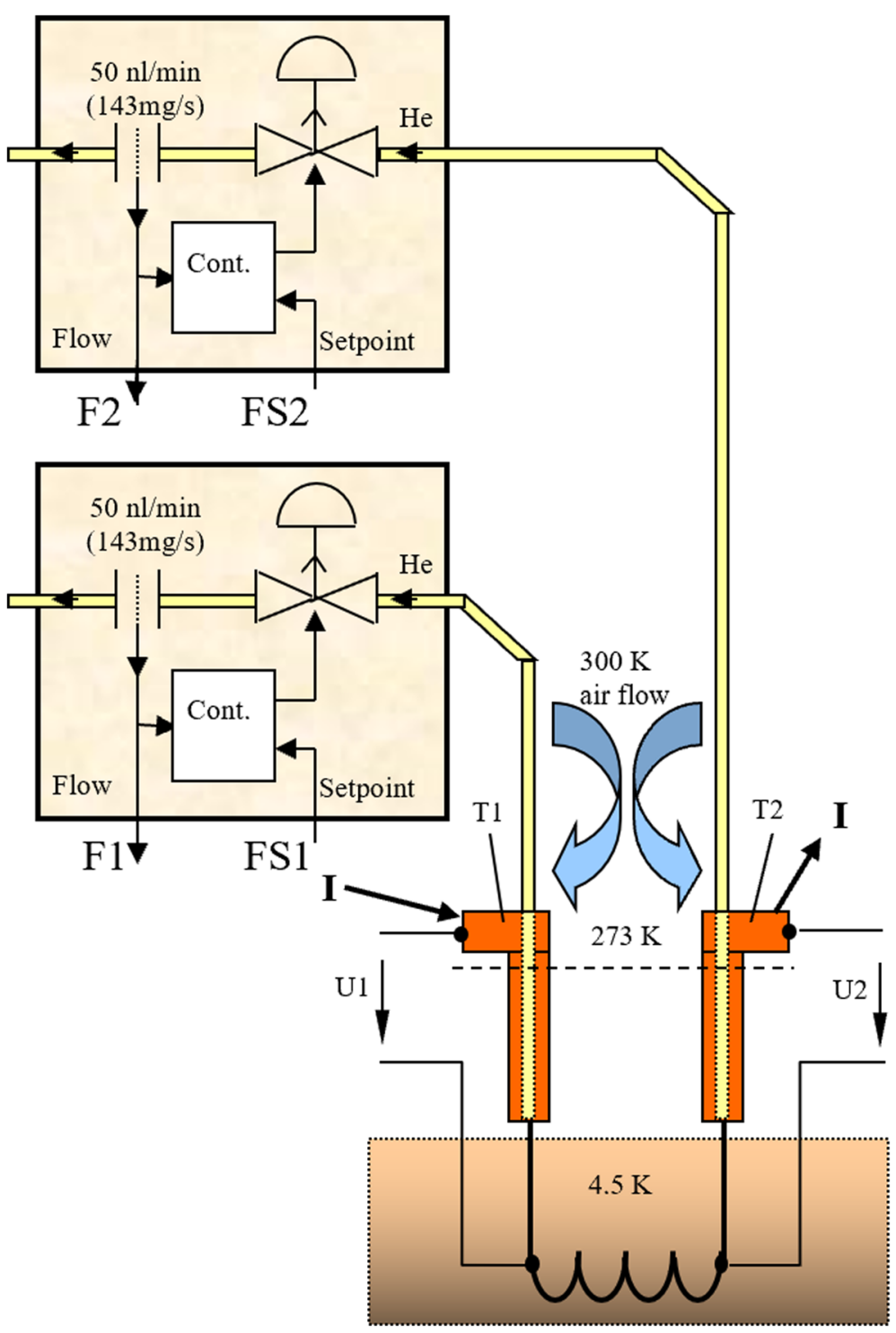

Fig. 12 The current lead control of SSM and SCQ

$273 \mathrm{~K}$ at exiting the lead; Helium temperature must reach the room temperature when passing control valve. Figure 12 illustrates an example for the cooling of a pair of current leads.

Formula for gas flow and lead voltage is as follows,

$$
\dot{m}= \begin{cases}d & U \leq 5 m V \\ a * U^{2}+b+c * \frac{\mathrm{du}}{\mathrm{d} t} & U>5 m V\end{cases}
$$

Where $\dot{m}$ is the helium gas flow; $\mathrm{U}$ is the voltage of current leads; a, b, c and d are empirical coefficients. 

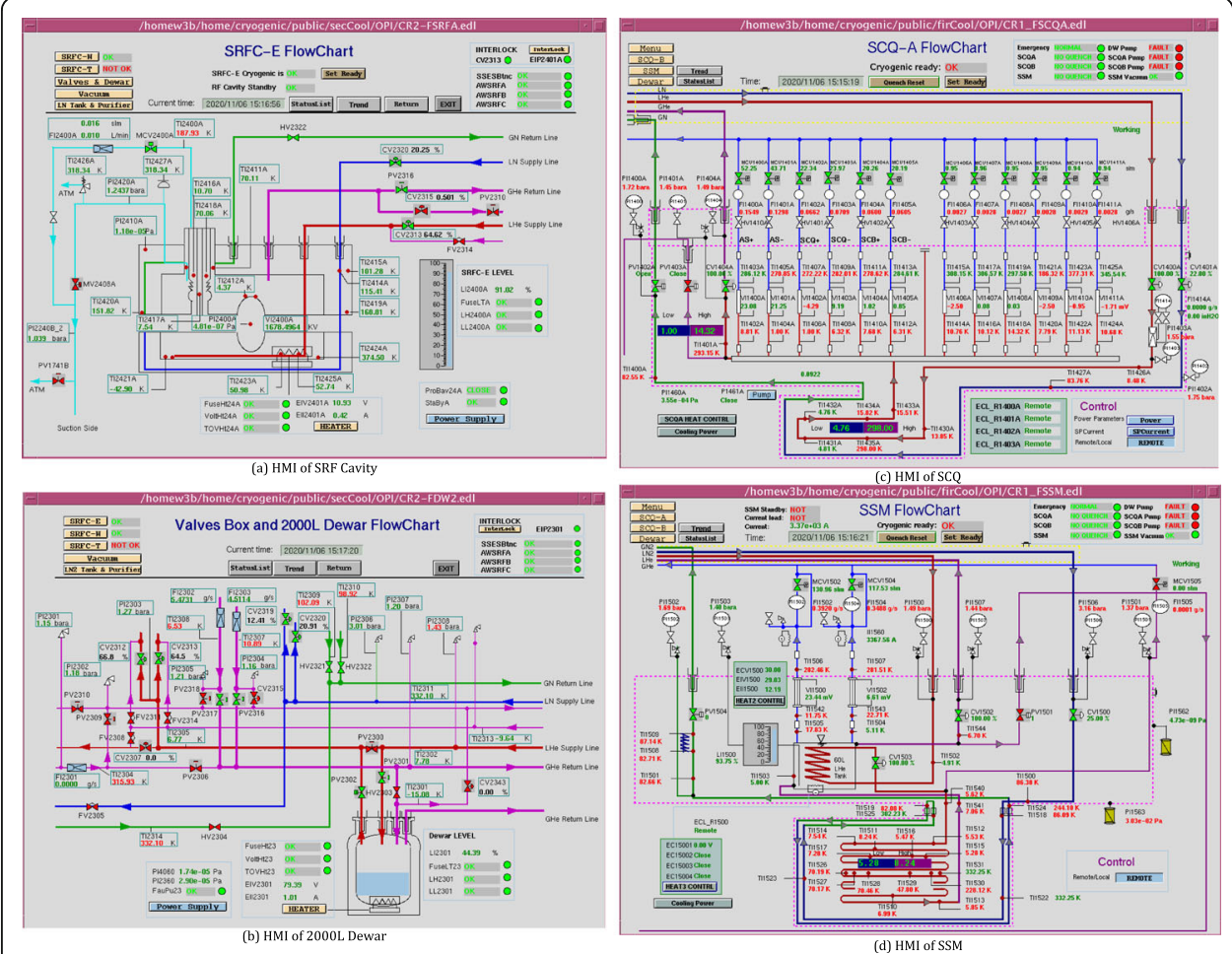

Fig. 13 The main HMls of cryogenic control system

\section{Human machine Interface}

For easy operation, the human machine interface (HMI) is designed and developed with user-friendly EDM (Extensible Display Manager) (https://www.slac.stanford.edu/grp/ $\mathrm{cd} /$ soft/epics/extensions/edm/edm.html) Figure 13 shows the several main HMIs of SRF cavity, 2000 L Dewar, SCQ and SSM in the cryogenic control system. The monitoring and control of cryogenic equipment are interactively operated by HMI. If parameters such as liquid level or pressure need to be adjusted, one could click the corresponding widget on the main HMI to modify them.

\section{Commissioning and operation of SRF cavities}

After the cryogenic control system of SRF cavity was delivered and put into operation, the cryogenic system of SRF cavity began to cool down in early 2006. On March 16th of the same year, the test team conducted on-site test and acceptance. The test results showed that the cryogenic system of SRF cavity could meet the working requirements of SRF cavity, marking that the commissioning of cryogenic system of SRF cavity was basically completed. After completing the dummy load, the off-line and online cryogenic tests, acceptance tests of the cryogenic systems of the east and west SRF cavities in Nov of the same year indicated that the construction of the cryogenic system of the SRF cavity was completed and officially put into operation. The cryogenic system on the SRF cavity side fully meets the operating requirements of the two SRF cavities simultaneously. In the 14 years since then, cryogenic system of the SRF cavity has been 


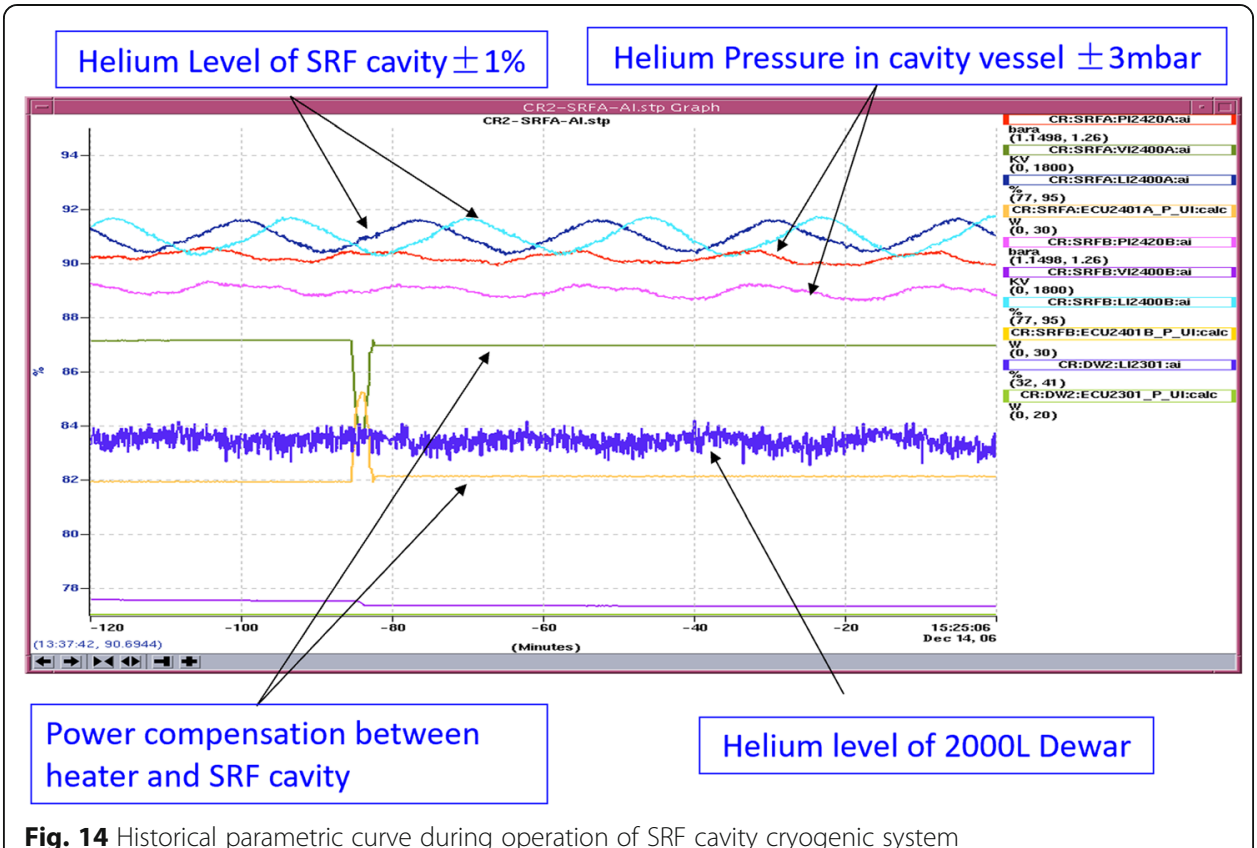

Fig. 14 Historical parametric curve during operation of SRF cavity cryogenic system

operating stably. Figure 14 shows the parametric curve during operation of SRF cavity cryogenic system.

\section{Joint test and commissioning of SC magnets}

Three BEPCII superconducting magnets are located at the first collision zone of the storage ring, and SCQs are symmetrically installed in SSM detector. In May 2007, a joint cooling test of three superconducting magnets was carried out, and the three magnets reached the superconducting state at the same time on May 11, 2007. On June 10, 2007, the SSM magnet and SCQA/B magnets were ramped to fully current at the same time, which indicates that the cryogenic system at the side of superconducting magnets

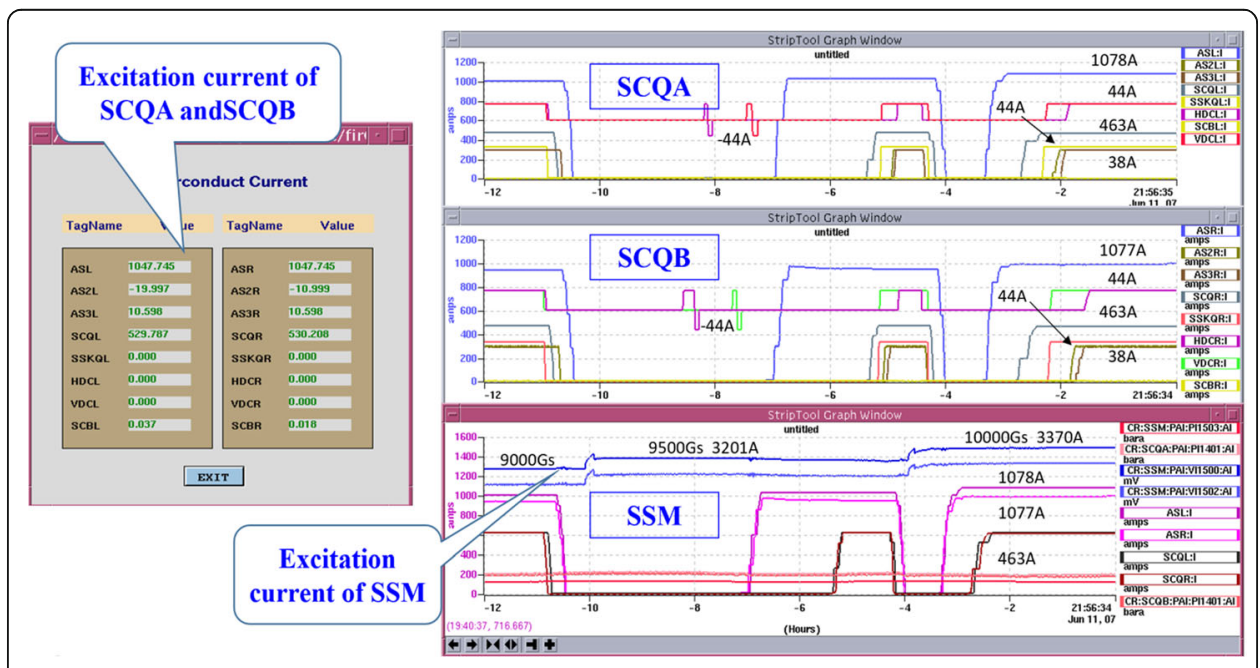

Fig. 15 Historical parametric curve during operation of SSM and SCQ cryogenic system 


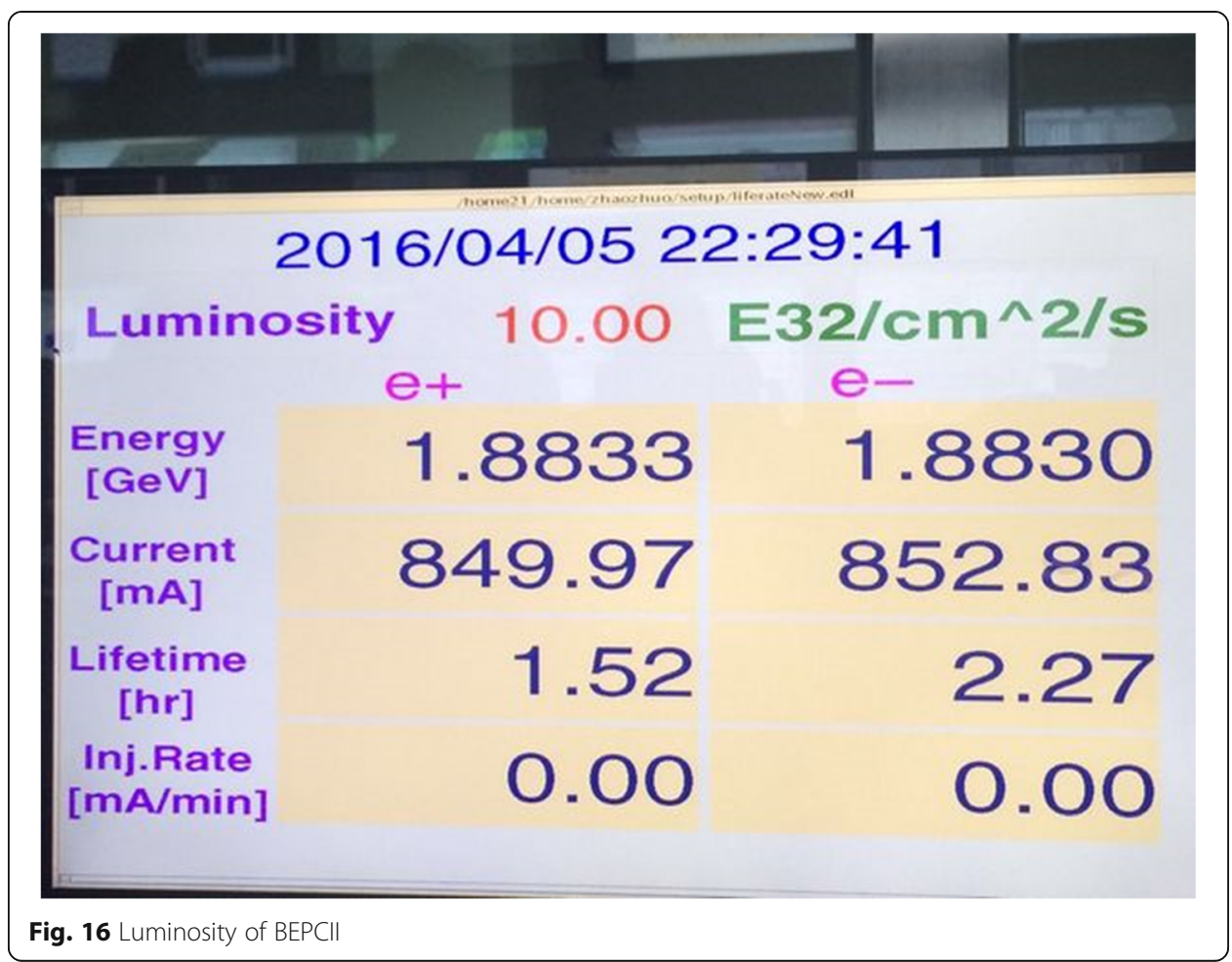

can fully meet the operation requirements of superconducting magnets. Figure 15 shows the parametric curve during operation of SSM and SCQ cryogenic system. So far, the construction of the entire cryogenic system and cryogenic control system had been completed.

\section{Conclusion}

The BEPCII cryogenic control system is divided into two parts. The refrigerator is controlled by Siemens PLC S7. "EPICS-IOC + PLC" double layer control structure is adopted in the superconducting device control system. The main control program is running in EPICS IOC, where control algorithms, PID control loops and sequences reside. While, Allen-Bradley PLC acts as I/O channels and runs the important and necessary interlocks. Since the cryogenic control system was put into operation at the end of 2005, it had been running stably. To unify the control system, it is planned to integrate the refrigerator control into the EPICS system. At 22:29 pm on April 5, 2016, BEPCII Luminosity reached $1 \times 10^{33} \mathrm{~cm}^{-2} \mathrm{~s}^{-1}$, seeing Fig. 16. The authors would like to to express their gratitude to Mr. Matthias Clausen and Dr. Holger Lierl from DESY, who had given a lot of valuable advice and help, during the design and commissioning of BEPCII cryogenic control system. People who contribute to the BEPCII cryogenic control system are also acknowledged. 
Author's contributions

GL conceived of the presented idea and wrote the manuscript with support from RY, MS, SL, ZZ, JZ, RH, GL and RG. All authors discussed the results and contributed to the final manuscript. The author(s) read and approved the final manuscript.

Funding

Not applicable.

Availability of data and materials

Not applicable.

Competing interests

The authors declare they have no competing interests.

Received: 29 December 2020 Accepted: 3 March 2021

Published online: 13 April 2021

References

1. Chuang Z. Introduction of the Project BEPCII. Beijing: MAC05; 2005.

2. Ji-Jiu Z, et al. BEPCII Control System. High Energy Phys Nucl Phys. 2006;30:11.

3. Matthias C. Review summary. Beijing: BEPCII cryogenic control Seminar; 2005.

\section{Publisher's Note}

Springer Nature remains neutral with regard to jurisdictional claims in published maps and institutional affiliations.

Submit your manuscript to a SpringerOpen ${ }^{0}$ journal and benefit from:

- Convenient online submission

- Rigorous peer review

- Open access: articles freely available online

- High visibility within the field

- Retaining the copyright to your article

Submit your next manuscript at $>$ springeropen.com 